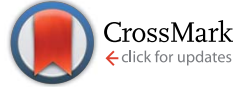

Cite this: RSC Adv., 2015, 5, 63691

Received 13th April 2015 Accepted 20th July 2015

DOI: $10.1039 / c 5 r a 06635 b$

www.rsc.org/advances

\section{Valorization of lignin waste from hydrothermal treatment of biomass: towards porous carbonaceous composites for continuous hydrogenation $\dagger$}

\author{
Gianpaolo Chieffi, Nina Fechler and Davide Esposito*
}

\begin{abstract}
Alkali lignin has been accumulated as a by-product mixed with barium salts during the hydrothermal treatment of rye straw with $\mathrm{Ba}(\mathrm{OH})_{2}$. Direct heat treatment followed by acid washing of such mineralized lignin were performed in order to obtain a porous material that was further exploited for the synthesis of a carbonaceous supported FeNi nanoparticle composite as active catalysts for continuous hydrogenation.
\end{abstract}

\section{Introduction}

Lignin is one of the most abundant organic polymers on our planet and the third major component of lignocellulosic biomass. ${ }^{1}$ Commercially, lignin is mostly obtained as the side product of cellulose pulp mills. ${ }^{2}$ Different pre-treatment processes that enable the isolation of the lignin fraction from the biomass have been reported. ${ }^{3}$ These are usually classified in accordance to the technology used and, among others, solvent fractionation (e.g. organosolv process), physical treatment (e.g. ball milling), biological treatment or chemical pre-treatment (oxidative, acidic or alkaline) have received great attention. Basic reagents such as sodium, calcium or ammonium hydroxide have been used for the effective chemical delignification of biomass. ${ }^{4}$ Such treatments favor to a good extent the cleavage of $\alpha$ - and $\beta$-ether linkages, producing a so-called alkali lignin mainly composed of oligomers in the range of 1-5 kDa.

Lignin has been recently used for the production of activated carbon (AC) by thermal treatment in the presence of alkali metal hydroxides. ${ }^{5,6}$ For instance, Fierro et al. studied the optimization of AC manufacture from Kraft lignin (KL) using $\mathrm{NaOH}$ or $\mathrm{KOH}$ as the activating agents. ${ }^{7}$ Wang et al. used a two-step procedure involving the pre-carbonization of the lignin, which is successively mixed with $\mathrm{KOH}$ before undergoing an

Max-Planck-Institute of Colloids and Interfaces, 14424 Potsdam, Germany. E-mail: davide.esposito@mpikg.mpg.de

$\dagger$ Electronic supplementary information (ESI) available. See DOI: $10.1039 / \mathrm{c} 5 \mathrm{ra} 06635 \mathrm{~b}$ additional heat-treatment. Final washing with $1 \mathrm{~N} \mathrm{HCl}$ provided porous carbon with very high surface area $\left(\sim 3000 \mathrm{~m}^{2} \mathrm{~g}^{-1}\right)$, which was successfully tested as an efficient Ni(II) adsorbent from wastewater. ${ }^{8}$

Metal loaded lignin has been also used as precursor for the preparation of supported nanoparticles (NPs). Mesoporous activated carbons, obtained by chemical activation of KL with $\mathrm{H}_{3} \mathrm{PO}_{4}$, were employed as supports for the synthesis of a carbonbased Pd material. The composites displayed activity for a broad range of transformations including the oxidation of toluene and xylene, ${ }^{9}$ selected example of hydrogenations and the SuzukiMiyaura cross-coupling. ${ }^{10}$

Besides the use of rare and expensive metals, composites based on iron, the fourth most abundant element in the lithosphere, have also been prepared. In this regard, Rodriguez et al. used $\mathrm{FeCl}_{3}$ both as the activating agent for lignin isolated from alkaline-pulping black liquors as well as precursor for the introduction of stable $\mathrm{Fe}$ nanoparticles in the carbonaceous matrix. This system was used as catalyst in wet peroxide oxidation of phenol. ${ }^{11}$ Interestingly, carbonaceous supported FeNi NPs have also proved to be an efficient hydrogenation catalyst for a variety of substrates. ${ }^{12-14}$

Recently, our group developed an efficient alkaline hydrothermal treatment of biomass e.g., rye straw, which affords the platform chemical lactic acid in high yields. ${ }^{15,16}$ The method relies on the use of barium hydroxide excess and generates alkali lignin as a by-product. The latter can be recovered in the form of a mixture with barium inorganic salts.

In order to further increase the value chain and inspired by other groups that reported on the use of carbonaceous residues from biomass for the preparation of heterogeneous catalysts or chromatographic supports, ${ }^{17-19}$ we here utilized the waste stream of the alkaline hydrothermal digestion of biomass, i.e. mineralized lignin, as direct "all-in-one" starting material for the preparation of porous carbonaceous material where the inherently included barium salts are used as porogen. ${ }^{20-23}$

The obtained materials were then used as support for ironnickel metal nanoparticles which exhibited remarkable 
catalytic activities for the continuous flow hydrogenation of nitrobenzene and phenylacetylene, commonly reduced using scarce and expensive noble metal supported catalysts. ${ }^{24}$

\section{Result and discussion}

The first step of our process was the hydrothermal treatment of biomass (rye straw) using $\mathrm{Ba}(\mathrm{OH})_{2}$, which despite its toxicity compared to other alkaline compounds, is known to promote the formation of lactic acid in high yields, ${ }^{15}$ and led to the recovery of a precipitate containing lignin-derived oligomers (see the ESI $\dagger$ ). In this study, we focused on the valorization of this otherwise waste crude by-product consisting in a lignin/ barium salt mixture (ML: mineralized lignin) as "all-in-one" precursor for porous carbonaceous materials. Initially, ML, which precipitates after the hydrothermal process, could be easily obtained by filtration (Scheme 1, step 1).

The X-ray diffraction (XRD) pattern of the crude filter cake revealed the typical diffraction peaks of barium carbonate (Fig. 1a). Carbonates are classical by-products of alkali hydrothermal treatments of biomass. ${ }^{25}$ The crystallite size of the salt was calculated by Scherrer equation to be approx. $26 \mathrm{~nm}$ in diameter. The presence of this compound was additionally confirmed by comparison of the IR spectrum of the ML (Fig. 2a) with the one of the pure $\mathrm{BaCO}_{3}$ (Fig. 2b). The absorption bands at $400-1800 \mathrm{~cm}^{-1}$, also visible in $\mathrm{ML}$, were attributed to vibrations of $\mathrm{CO}_{3}{ }^{2-}$ and reflect the asymmetric $\mathrm{C}-\mathrm{O}$ stretching $\left(1427 \mathrm{~cm}^{-1}\right)$, out of plane bending $\left(856 \mathrm{~cm}^{-1}\right)$ and in plane bending vibration $\left(692 \mathrm{~cm}^{-1}\right) .{ }^{26}$

Besides $\mathrm{BaCO}_{3}$, the IR spectrum of ML also confirmed the presence of some characteristic infrared bands attributed to lignin: a broad band at $3340 \mathrm{~cm}^{-1}$ corresponding to the $\mathrm{O}-\mathrm{H}$ of alcoholic and phenolic groups stretching vibrations, and the shoulder at $1644 \mathrm{~cm}^{-1}$ caused by the stretching of carbonyl groups conjugated with aromatic rings. Aromatic skeleton vibration was also visible and appeared at $1594 \mathrm{~cm}^{-1} \cdot{ }^{27}$

Elemental analysis of the ML (see Table $\mathrm{S} 1 \dagger$ ) revealed a residual carbon content of $\sim 17 \mathrm{wt} \%$ and the relative TGA analysis under inert atmosphere (see Fig. S1†) showed a weight loss of $38 \%$ at $1000{ }^{\circ} \mathrm{C}$ associated with release of volatiles. ${ }^{28}$

At this stage, the organic-inorganic solid ML was directly used as carbonaceous precursor and heat-treated in a nitrogen oven at $800{ }^{\circ} \mathrm{C}$ for $2 \mathrm{~h}$ where $\mathrm{BaCO}_{3}$ served as the in situ

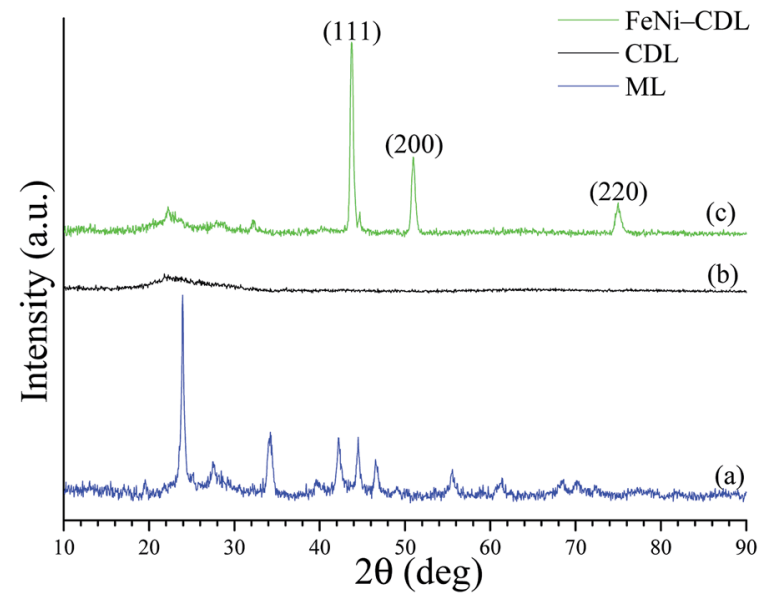

Fig. 1 XRD patterns of the mineralized lignin (a), of the carbonized/ demineralized lignin (b) and of the carbonized/demineralized lignin impregnated with the salt precursors of $\mathrm{Fe}$ and $\mathrm{Ni}$ and then heattreated at $800^{\circ} \mathrm{C}$ for $2 \mathrm{~h}$ under $\mathrm{N}_{2}$ (c).

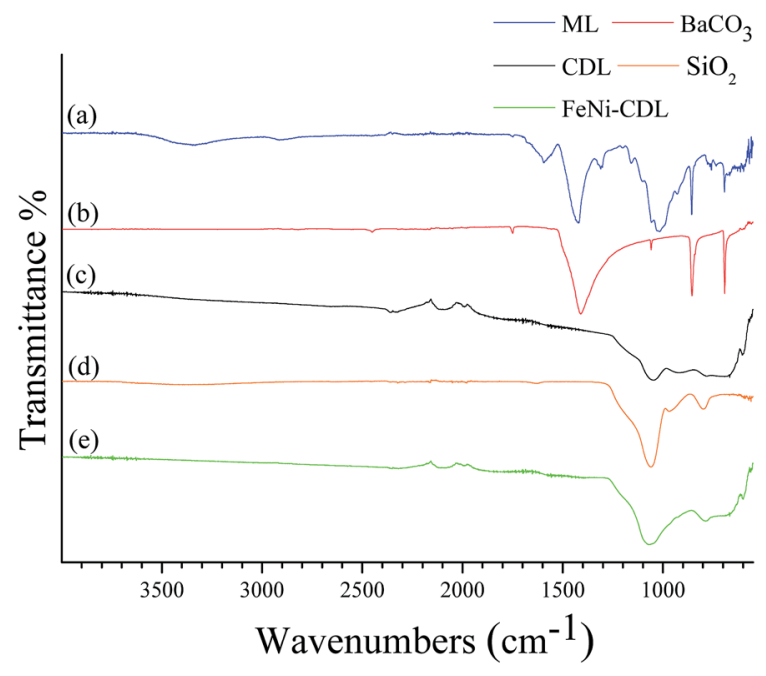

Fig. 2 IR spectra of the mineralized lignin (a), of $\mathrm{BaCO}_{3}(\mathrm{~b})$, of the carbonized/demineralized lignin (c), of $\mathrm{SiO}_{2}$ (d), and of the carbonized/ demineralized lignin impregnated with the salt precursors of Fe and $\mathrm{Ni}$ and then heat-treated at $800^{\circ} \mathrm{C}$ for $2 \mathrm{~h}$ under $\mathrm{N}_{2}$ (e)

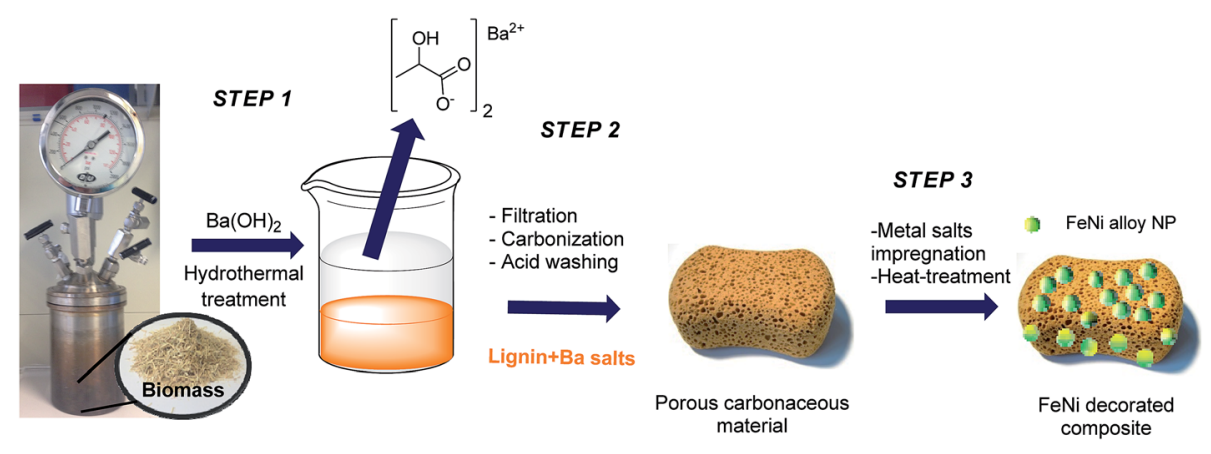

Scheme 1 Process chain for the synthesis of porous lignin derived carbonaceous material and the respective supported FeNi NPs composite. 
activation and porogen agent. The carbonized product was further washed with diluted hydrochloric acid in order to remove the inorganic content and free the pores of the resulting carbonaceous material (CDL: carbonized demineralized lignin), which was isolated as a black powder in $16 \mathrm{wt} \%$ yield after simple vacuum drying (Scheme 1, step 2). The removal of crystalline components was confirmed by XRD (Fig. 1b). However, IR revealed the presence of an adsorption band centered at $1060 \mathrm{~cm}^{-1}$, which could be ascribed to the asymmetric bending modes of $\mathrm{SiO}_{2}$, pointing to residual amounts of silica (see Fig. 2c and d) commonly found in straw ashes..$^{29,30}$ Scanning electron microscopy (SEM) revealed a different morphological structure for crude ML (Fig. 3a and $\mathrm{a}^{\prime}$ ) compared to CDL (Fig. 3b and $\mathrm{b}^{\prime}$ ).

The crude ML material possessed a flake-like morphology with extended thick sheets over several microns. In contrast, CDL showed much finer structures which suggested the successful removal of the barium salts thus giving access to the actual carbonaceous material. In order to assess the porosity in more detail, nitrogen sorption measurements were performed (Fig. $4 \mathrm{a}$ and b).

Here, the Brunauer-Emmett-Teller (BET) calculation for ML gave a negligible surface area of $11 \mathrm{~m}^{2} \mathrm{~g}^{-1}$ whereas the final carbonaceous material CDL showed an increased surface area of $539 \mathrm{~m}^{2} \mathrm{~g}^{-1}$ confirming the assumptions from the SEM images. Furthermore, the isotherm of CDL displayed high nitrogen uptake already at low relative pressures indicative for a reasonable amount of micropores. The isotherm of CDL shows a type IV behavior which is usually ascribed to the presence of mesopores. Average pore size was calculated from the nitrogen sorption isotherms using the NLDFT model for slit pores to be of $5 \mathrm{~nm}$ in diameter (see Fig. $\mathrm{S} 2 \dagger$ ). Here, it is to be mentioned that this size is much smaller than the barium carbonate crystallite size obtained from XRD measurements which is attributed to the deviating properties of the carbon described here from the model on which calculations are based as well as to cavitation effects.

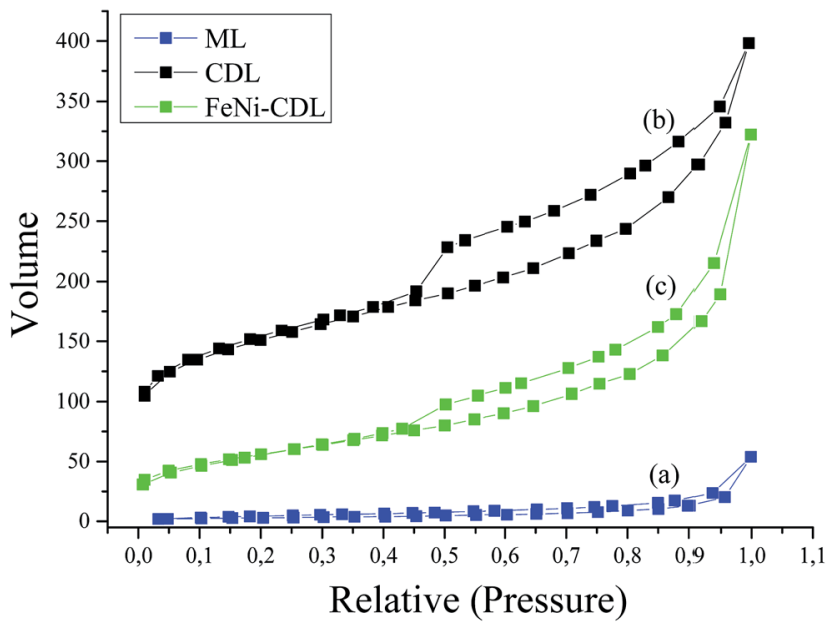

Fig. 4 Nitrogen sorption isotherms of the ML (a), CDL (b) and FeNiCDL (c).

Eventually, the direct heat treatment of ML followed by acid washing could be exploited for the synthesis of high surface area carbonaceous materials with micro- and mesoporosity. Such features are highly desirable for catalytic reactions where high mass transport is required.

With this is mind, the CDL was thus considered as a catalyst precursor and further functionalized with iron nickel nanoparticles (FeNi-CDL). This was achieved by impregnation of CDL with an alcoholic solution of the respective metal nitrate salts followed by heat-treatment at $800{ }^{\circ} \mathrm{C}$ for $2 \mathrm{~h}$ under $\mathrm{N}_{2}$ atmosphere (Scheme 1, step 3). This yielded a fine black powder and the XRD confirmed the formation of a fcc crystalline phase of a FeNi alloy with a NP crystallite size of $17 \mathrm{~nm}$ (Fig. 1c). ${ }^{31}$ Besides the main phase, an additional less intense peak found at $44.75 \theta$ revealed the presence of a concomitant bcc phase of the same alloy. ${ }^{32}$ The morphology of the FeNi-CDL resembled quite well the one of the
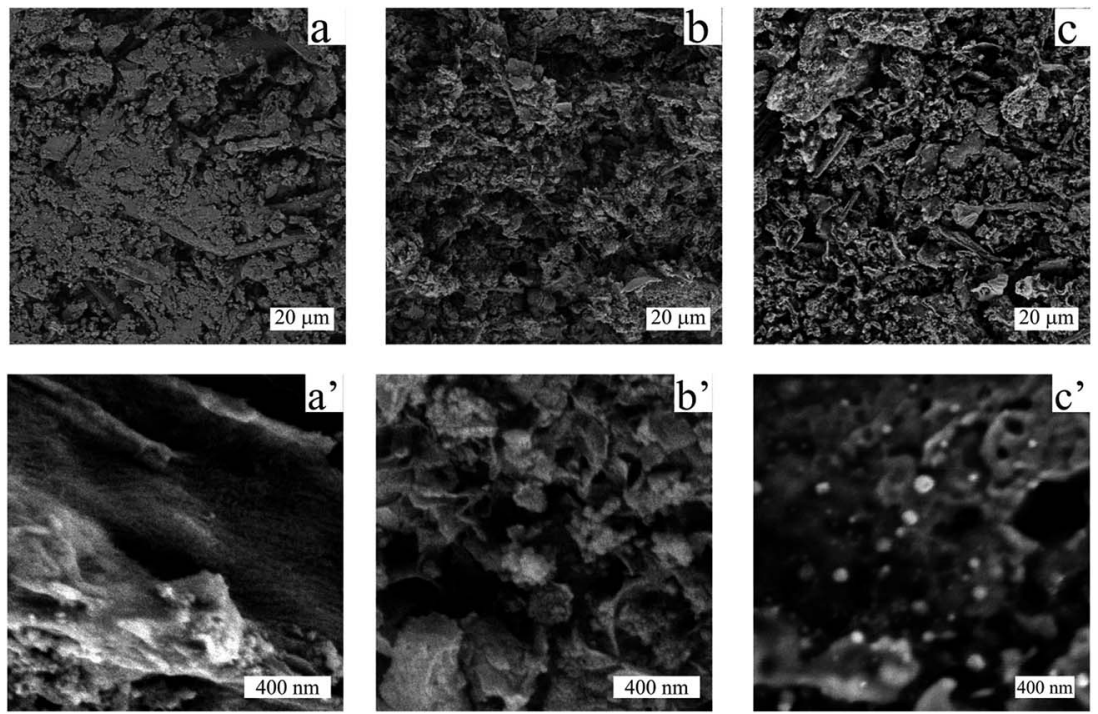

Fig. 3 Low magnification $(a-c)$ and high magnification $\left(a^{\prime}-c^{\prime}\right)$ SEM images of the ML (a and $\left.a^{\prime}\right), C D L\left(b\right.$ and $\left.b^{\prime}\right)$ and FeNi-CDL $\left(c\right.$ and $\left.c^{\prime}\right)$. 

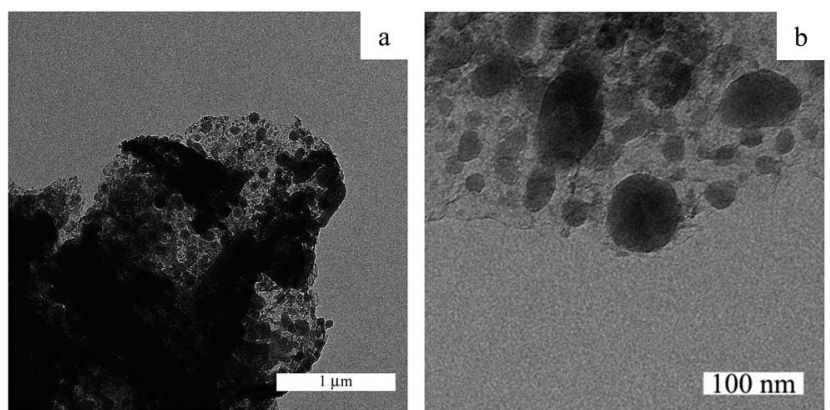

Fig. 5 Low (a) and high (b) magnification TEM images of the carbonized/demineralized lignin impregnated with the salt precursors of $\mathrm{Fe}$ and $\mathrm{Ni}$ and then heat-treated at $800{ }^{\circ} \mathrm{C}$ for $2 \mathrm{~h}$ under $\mathrm{N}_{2}$.

original carbonaceous material, however, well visible bright metal nanoparticles with an average size of $48 \mathrm{~nm}$ in diameter could be detected (Fig. $3 \mathrm{c}$ and $\mathrm{c}^{\prime}$ ). The presence of iron and nickel was confirmed by EDX analysis (see Fig. S3 in the ESI $\dagger$ ).

TEM analysis of FeNi-CDL revealed a relatively broad size distribution and the coexistence of metal particle conglomerates in the micrometer range, together with round shape nanoparticles embedded in the carbonaceous matrix (see Fig. 5a and b). The dispersed non-aggregated nanoparticles visible at high magnification showed an average size distribution of $48 \mathrm{~nm}$ (the size distribution graph is reported in the ESI in Fig. S4 $\dagger$ ). The fact that the mean particle size observed from both SEM and TEM images was larger than the average crystallite size calculated using the Scherrer equation from the XRD pattern $(17 \mathrm{~nm})$ confirmed that these nanoparticles were partly conglomerates.
Also nitrogen sorption analysis still revealed an isotherm shape similar to the one of the initial CDL, i.e. particles are deposited on the outer surface, pointing to the successful functionalization of CDL under preservation of the porosity as well as morphology. The somewhat lowered surface area of $198 \mathrm{~m}^{2} \mathrm{~g}^{-1}$ could be ascribed to the increased mass of the additional metals (Fig. $4 \mathrm{c}$ ).

Inductively coupled plasma-optical emission spectroscopy (ICP-OES) analysis of FeNi-CDL displayed the simultaneous presence of $\mathrm{Fe}(14 \mathrm{wt} \%)$ and $\mathrm{Ni}(22 \mathrm{wt} \%)$ in the initially employed ratio of the metal salt precursors as well as residues of $\mathrm{Ba}$ and Si from the ML (see Table S1 $\dagger$ ). As mentioned earlier, the focus of the present contribution was more the facile and sustainable valorization of the alkali hydrothermal process chain value. Therefore, a standardized washing procedure was applied without optimizing conditions for a full removal of inorganic impurities. Moreover, as it will be discussed in the following, the residual presence of $\mathrm{Si}$ and Ba was not detrimental for the catalytic performances of the FeNi-CDL, and possible additional effects caused by these inorganic impurities will be evaluated in future studies.

Having established the protocol for the preparation of FeNiCDL from the alkali lignin, we assessed the catalytic performance of such material for hydrogenation reactions. For this purpose, the FeNi-carbonaceous composite powder was packed into a cartridge and directly employed as a catalyst within a bench-top continuous flow reactor (H-Cube Pro $^{\mathrm{TM}}$ ) provided with an internal $\mathrm{H}_{2}$ source and equipped with a liquid feed.

Table 1 reports the conversion and selectivity for the hydrogenation of two different model substances. The flow rate and the molarity of the different substrates were kept constant

Table 1 Conversion, selectivity and optimized temperature and pressure for model continuous hydrogenation reactions using the FeNi-CDL as catalyst

Entry

\footnotetext{
${ }^{a}$ Conditions: $50 \mathrm{mM}$ in ethanol, $0.3 \mathrm{~mL} \mathrm{~min}{ }^{-1} \cdot{ }^{b} \mathrm{CDL}$ was used as catalyst. ${ }^{c} 100 \mathrm{mM}, 0.5 \mathrm{~mL} \mathrm{~min}{ }^{-1} \cdot{ }^{d}$ Lindlar catalyst. ${ }^{e}$ Liquid feed compositions are expressed as uncorrected GC/MS peak area percentages.
} 
(50 $\mathrm{mM}$ and $0.3 \mathrm{~mL} \mathrm{~min}^{-1}$ ), while temperature and hydrogen pressures were varied to optimize conversion and selectivity.

The catalytic hydrogenation of nitroaromatic compounds is a crucial reaction for the production of anilines, which represent an important intermediate for the synthesis of pharmaceuticals, polyurethanes, dyes and agricultural products. ${ }^{33,34}$ As a model for such transformation, the reduction of nitrobenzene was evaluated. The latter compound could be quantitatively converted into aniline (entry 1) at $125{ }^{\circ} \mathrm{C}$ and 20 bar of $\mathrm{H}_{2}$. The GC chromatograms and the corresponding mass spectra of the major products from Table 1 are reported in Fig. S5 and S6 in the ESI. $\dagger$

Control experiments performed with the non-functionalized CDL did not show effective hydrogenation of nitrobenzene under the same experimental conditions, confirming in a preliminary way that the presence of inorganic residues inherently present from the staring material was negligible with regard to the catalytic performance and underlined the active role of FeNi NPs in the catalytic cycle (entry 2).

Phenylacetylene was also tested as substrate for the continuous hydrogenation in order to prove the versatility of the FeNiCDL for the reduction of additional functional groups (entry 3). The complete conversion of the starting material at high selectivity toward ethylbenzene was achieved at $150{ }^{\circ} \mathrm{C}$ and 50 bar of $\mathrm{H}_{2}$.

Interestingly, the selectivity of the reaction could be tuned towards styrene at milder conditions (entry 4) and increasing the molarity $(100 \mathrm{mM})$ as well as the flow rate $\left(0.5 \mathrm{~mL} \mathrm{~min}^{-1}\right)$. Traditionally, similar processes are performed using commercial catalysts as the Lindlar, a palladium catalyst poisoned with traces of lead and quinoline. ${ }^{35}$ Nevertheless, using the latter under the same experimental conditions (entry 5), ethylbenzene was obtained. The selective hydrogenation to styrene could not be achieved even when the reaction was performed at room temperature and with 1 bar of $\mathrm{H}_{2}$ (entry 6), revealing our FeNiCDL composite as an interesting candidate for selective hydrogenation processes.

\section{Conclusions}

In this work we showed how the integrated processes for the smart recycle and upcycle of biomass derived waste streams could be efficiently combined with strategies for the preparation of functional carbonaceous and composites materials based on cheap and abundant metal precursors of iron and nickel. Porous carbonaceous materials were obtained via the heat-treatment of mineralized lignin isolated from the waste stream of the alkaline hydrothermal digestion of rye straw. The $\mathrm{BaCO}_{3}$ contained in the "all-in-one" crude lignin carbonaceous precursor could be in this way upcycled and served as an efficient in situ activation and poration agent, leading to the formation of porous carbonaceous materials which were further functionalized with FeNi alloys nanoparticles. The resulting nanocomposite displayed remarkable catalytic activity for the flow hydrogenation of nitrobenzene and phenylacetylene to aniline and ethylbenzene, respectively. Moreover the FeNi-CDL was effective for the selective partial hydrogenation of phenylacetylene to styrene under mild conditions without the use of any additives, unlike the commercial Lindlar catalyst. Therefore, the approach described in this work demonstrates nicely the efficient valorization of a waste stream from an independent biomass conversion scheme and opens the way to a fully integrated biorefinery with increased chain value. Future studies will focus on the preparation of analogs of this novel catalytic system and the evaluation of their activity for additional catalytic transformations. Moreover, the possible synergistic effects of the inorganic residues contained in the waste biomass on the overall catalytic performance of the final composites will be further evaluated.

\section{Acknowledgements}

The authors gratefully thank Hendrik Wetzel for ICP OES measurements (Fraunhofer Institute for Applied Polymer Research).

\section{Notes and references}

1 D. M. Alonso, S. G. Wettstein and J. A. Dumesic, Chem. Soc. Rev., 2012, 41, 8075-8098.

2 J. Lora and W. Glasser, J. Polym. Environ., 2002, 10, 39-48.

3 L. da Costa Sousa, S. P. S. Chundawat, V. Balan and B. E. Dale, Curr. Opin. Biotechnol., 2009, 20, 339-347.

4 Y. C. Park and J. S. Kim, Energy, 2012, 47, 31-35.

5 Y. Zhai, Y. Dou, D. Zhao, P. F. Fulvio, R. T. Mayes and S. Dai, Adv. Mater., 2011, 23, 4828-4850.

6 L. L. Zhang and X. S. Zhao, Chem. Soc. Rev., 2009, 38, 25202531.

7 V. Fierro, V. Torné-Fernández and A. Celzard, Microporous Mesoporous Mater., 2007, 101, 419-431.

8 Y. Gao, Q. Yue, B. Gao, Y. Sun, W. Wang, Q. Li and Y. Wang, Chem. Eng. J., 2013, 217, 345-353.

9 J. Bedia, J. M. Rosas, J. Rodríguez-Mirasol and T. Cordero, Appl. Catal., B, 2010, 94, 8-18.

10 E. Guillén, R. Rico, J. M. López-Romero, J. Bedia, J. M. Rosas, J. Rodríguez-Mirasol and T. Cordero, Appl. Catal., A, 2009, 368, 113-120.

11 J. A. Zazo, J. Bedia, C. M. Fierro, G. Pliego, J. A. Casas and J. J. Rodriguez, Catal. Today, 2012, 187, 115-121.

12 P. Gallezot, P. J. Cerino, B. Blanc, G. Flèche and P. Fuertes, J. Catal., 1994, 146, 93-102.

13 S. Sitthisa, W. An and D. E. Resasco, J. Catal., 2011, 284, 90101.

14 G. Chieffi, C. Giordano, M. Antonietti and D. Esposito, J. Mater. Chem. A, 2014, 2, 11591-11596.

15 D. Esposito and M. Antonietti, ChemSusChem, 2013, 6, 989992.

16 G.-Q. Chen and M. K. Patel, Chem. Rev., 2012, 112, 20822099.

17 R. Luque, A. Pineda, J. C. Colmenares, J. M. Campelo, A. A. Romero, J. C. Serrano-Riz, L. F. Cabeza and J. CotGores, J. Nat. Gas Chem., 2012, 21, 246-250. 
18 M.-M. Titirici, R. J. White, N. Brun, V. L. Budarin, D. S. Su, F. del Monte, J. H. Clark and M. J. MacLachlan, Chem. Soc. Rev., 2015, 44, 250-290.

19 R. J. White, V. Budarin, R. Luque, J. H. Clark and D. J. Macquarrie, Chem. Soc. Rev., 2009, 38, 3401-3418.

20 P. J. M. Suhas, M. M. L. Carrott and R. Carrott, Lignin - from natural adsorbent to activated carbon: A review, Bioresour. Technol., 2007, 98(12), 2301-2312.

21 J. M. Dias, M. C. M. Alvim-Ferraz, M. F. Almeida, J. RiveraUtrilla and M. Sánchez-Polo, J. Environ. Manage., 2007, 85, 833-846.

22 C. Zhang, M. Antonietti and T.-P. Fellinger, Adv. Funct. Mater., 2014, 24, 7655-7665.

23 N. Fechler, T.-P. Fellinger and M. Antonietti, Adv. Mater., 2013, 25, 75-79.

24 (a) S. Kataoka, Y. Takeuchi, A. Harada, T. Takagi, Y. Takenaka, N. Fukaya, H. Yasuda, T. Ohmori and A. Endo, Appl. Catal., A, 2012, 427-428, 119-124; (b) J. Hu, Z. Zhou, R. Zhang, L. Li and Z. Cheng, J. Mol. Catal. A: Chem., 2014, 381, 61-69.
25 J. A. Onwudili and P. T. Williams, Int. J. Hydrogen Energy, 2009, 34, 5645-5656.

26 N. Tipcompor, T. Thongtem, A. Phuruangrat and S. Thongtem, Mater. Lett., 2012, 87, 153-156.

27 B. Xiao, X. F. Sun and R. Sun, Polym. Degrad. Stab., 2001, 74, 307-319.

28 S. Munir, S. S. Daood, W. Nimmo, A. M. Cunliffe and B. M. Gibbs, Bioresour. Technol., 2009, 100, 1413-1418.

29 A. E. Ghaly and A. Al-Taweel, Energy Sources, 1990, 12, 131145.

30 G.-M. Gao, H.-F. Zou, S.-C. Gan, Z.-J. Liu, B.-C. An, J.-J. Xu and G.-H. Li, Powder Technol., 2009, 191, 47-51.

31 Y. Chen, X. Luo, G.-H. Yue, X. Luo and D.-L. Peng, Mater. Chem. Phys., 2009, 113, 412-416.

32 Y. Liu, Y. Chi, S. Shan, J. Yin, J. Luo and C.-J. Zhong, J. Alloys Compd., 2014, 587, 260-266.

33 T. Stemmler, A.-E. Surkus, M.-M. Pohl, K. Junge and M. Beller, ChemSusChem, 2014, 7, 3012-3016.

34 X. Meng, H. Cheng, Y. Akiyama, Y. Hao, W. Qiao, Y. Yu, F. Zhao, S.-I. Fujita and M. Arai, J. Catal., 2009, 264, 1-10.

35 H. Lindlar and R. Dubuis, Org. Synth., 1966, 46, 89. 